\title{
Consensus Analysis of Second-Order Multiagent Systems with General Topology and Time Delay
}

\author{
Bo Liu, ${ }^{1}$ Guangming Xie, ${ }^{2,3}$ Yanping Gao, ${ }^{4}$ Jiaxi Wu, ${ }^{1}$ Jianguo Zhang, ${ }^{1}$ and Wenguang Luo ${ }^{5}$ \\ ${ }^{1}$ College of Science, North China University of Technology, Beijing 100144, China \\ ${ }^{2}$ Center for Systems and Control, Department of Mechanics and Engineering Science, Peking University, Beijing 100871, China \\ ${ }^{3}$ School of Electrical and Electronics Engineering, East China Jiaotong University, Nanchang 330013, China \\ ${ }^{4}$ College of Computer and Information Engineering, Beijing Technology and Business University, Beijing 100048, China \\ ${ }^{5}$ School of Electric and Information Engineering, Guangxi University of Science and Technology, Liuzhou 545006, China
}

Correspondence should be addressed to Guangming Xie; xiegming@pku.edu.cn

Received 27 November 2012; Revised 21 February 2013; Accepted 24 February 2013

Academic Editor: Vijay Gupta

Copyright (c) 2013 Bo Liu et al. This is an open access article distributed under the Creative Commons Attribution License, which permits unrestricted use, distribution, and reproduction in any medium, provided the original work is properly cited.

\begin{abstract}
This paper addresses the consensus of second-order multiagent systems with general topology and time delay based on the nearest neighbor rule. By using the Laplace transform technique, it is proved that the second-order multi-agent system in the presence of time-delay can reach consensus if the network topology contains a globally reachable node and time delay is bounded. The bound of time-delay only depends on eigenvalues of the Laplacian matrix of the system. The main contribution of this paper is that the accurate state of the consensus center and the upper bound of the communication delay to make the agents reach consensus are given. Some numerical simulations are given to illustrate the theoretical results.
\end{abstract}

\section{Introduction}

The wide applications of multiagent systems abounded in nature and engineering area have stimulated a great deal of interests in studying cooperative and coordinated control problems. The mechanisms operational principles of multiagent systems can provide useful ideas for developing distributed cooperative control, formation control, unmanned air vehicles, and sensing networks, and so forth. Recently, the study of collective dynamics and coordination of multiagent systems becomes a hot topic and has attracted many researchers from mathematics, physics, biology, sociology, control science, computer science, artificial intelligence, and so forth [1-25].

However, study of some fundamental issues concerning the coordinated control of multi-agent systems, such as consensus, stability, synchronization, and controllability analysis. As in its usual sense, consensus means multiple agents can reach an agreement on a common value by regulating their neighbors. The consensus problem was firstly studied by DeGroot [14], who proposed a weighted average method to estimate probability distribution function of unknown variables. Vicsek and Czirok [10] established a discrete model of the multi-agents system called "Vicsek Model." Jadbabaie et al. [11] developed "Vicsek Model," which had been linearized by graph theory and matrix theory. Usually, a multi-agent system is interconnected through neighbor rules and hence has a local and time-varying communication topology. In [4], Olfati-Saber and Murray discussed linear systems with timedelays.

Many papers on consensus problems of first-order multiagent system were presented (see, e.g., $[1-4,10,11,20,21]$ and the references therein). In [1], the first-order multiagent systems were firstly studied by the Laplace transform technique and it was proved that the system could reach consensus under certain conditions. In [2], the average consensus problem was investigated in multi-agent systems with undirected network topology and multiple time-varying communication delays by the linear matrix inequality (LMI) method. Liu et al. [23] considered the consensus of multiagent systems with an active leader. The second-order multiagent systems were studied in $[6,7,12,13,16-19,22]$. 
Yu et al. [6] discussed the second-order system with timedelays. They obtained the necessary and sufficient condition and gave upper bound of time-delays. In [7], Ren considered a consensus algorithm for second-order dynamics systems with Cartesian coordinate coupling in three-dimensional space. In [12], the authors discussed the consensus of continuous second-order multi-agent system in a sampled data set. Lin and Jia [22] investigated the consensus of a class of second-order multi-agent systems with time-delays and jointly connected topologies.

In the view of [1], the Laplace transform has many advantages in solving consensus problems, such as simplifying the model of first-order systems and obtaining the accurate state of the consensus center. Therefore, we will study the consensus of the second-order multi-agent system with timedelay and general topology by the Laplace transform in this paper. We will prove the consensus can be achieved when network topology contains a globally reachable node, and the time-delays must be less than a critical value which depends on the eigenvalues of the Laplacian matrix. Furthermore, the accurate state of the consensus center of the second-order multi-agent system and the upper bound of the communication delay to make the agents reach consensus are obtained.

The rest of this paper is organized as follows. In Section 2, we present the mathematical model of the second-order multi-agent system with time-delay and formulate it by matrix form. In Section 3, the detailed theoretical analysis for the model is given and the main results are derived. Then some numerical simulations are given to verify the theoretical results in Section 4. Finally, the conclusion is given in Section 5.

\section{Model}

Consider the second-order multi-agent system with timedelay and general topology is described by

$$
\begin{gathered}
\dot{x}_{i}=v_{i} \\
\dot{v}_{i}=-\sum_{j \in \mathcal{N}_{i}} a_{i j}\left(x_{i}(t-\tau)-x_{j}(t-\tau)\right)-v_{i},
\end{gathered}
$$

where $x_{i} \in \mathfrak{R}^{n}$ and $v_{i} \in \mathfrak{R}^{n}$ are position and velocity of agent $i$, respectively, and $i=1, \ldots, N ; \mathcal{N}_{i}$ is the neighbor set of agent $i ; A=\left[a_{i j}\right] \in \mathfrak{R}^{n \times n}(i, j=1, \ldots, N)$ with each $a_{i j} \geq 0$ and $a_{i i}=0$ is the interaction (or coupling) matrix; $\tau>0$ is the time-delay.

Let $X=\left(x_{1}, \ldots, x_{N}\right)^{T}$ and $V(t)=\left(v_{1}, v_{2}, \ldots, v_{N}\right)^{T}$ be the stack vector of all the agent states and velocities; it follows that

$$
\begin{gathered}
\dot{X}=V \\
\dot{V}=-L X(t-\tau)-V,
\end{gathered}
$$

where the matrix $L$ is the Laplacian and has the following properties: (i) the off-diagonal elements are all negative or zero, and (ii) the row sums are all equal to zero. And we can formulate the model with time-delay in the following form:

$$
\ddot{X}(t)+\dot{X}(t)=-L X(t-\tau) .
$$

For the Laplacian matrix $L$, we can have the following lemmas.

Lemma 1 (see [7]). Laplacian matrix L has at least one zero eigenvalue with $\mathbf{1}=(1,1, \ldots, 1)^{T}$ as its eigenvector, and all nonzero eigenvalues have positive real parts. Laplacian matrix $L$ has a simple zero eigenvalue if and only if $\mathscr{G}$ has a globally reachable node.

Lemma 2 (see [1]). For Laplacian matrix L, the algebraic complements of the elements in the same row are equal.

Lemma 3 (see [1]). For a column vector denoted as $z$, if a column of Laplacian matrix $L$ is replaced by $z$, the new matrix is denoted as $L_{1}$, and if another column of $L$ is replaced by $z$, the new matrix is denoted as $L_{2}$, then $\operatorname{det}\left(L_{1}\right)=\operatorname{det}\left(L_{2}\right)$.

\section{Main Results}

In this section, we will analyze the consensus of secondorder multi-agent systems with general topology and timedelay based on the nearest neighbor rule. For this, we use the Laplace transform technique into (3), then we can have

$$
\left[\left(s^{2}+s\right) I+e^{-s \tau} L\right] \mathscr{L}[X(t)]=s X(0)+X(0)+V(0),
$$

where $X(0)=\left(x_{1}(0), x_{2}(0), \ldots, x_{N}(0)\right)^{T}$ and $V(0)=$ $\left(v_{1}(0), v_{2}(0), \ldots, v_{N}(0)\right)^{T}$ are initial values.

For simplicity and without loss of generality, we will discuss the case $X(0)=0$ and $V(0) \neq 0$ throughout this paper.

Theorem 4. For second-order multi-agent system (1), if there exists at least one globally reachable node in the network topology, and the time-delay $\tau$ satisfies

$$
\begin{gathered}
\tau<\min _{k=\{2,3, \ldots, N\}}\left\{\frac{\left|\arccos \left(\left(\left\|\lambda_{k}\right\|-2\right) /\left\|\lambda_{k}\right\|\right)-\arg \left(\lambda_{k}\right)\right|}{\sqrt{\left\|\lambda_{k}\right\|-1}},\right. \\
\left.\frac{\pi / 2-\left|\arg \left(\lambda_{k}\right)\right|}{\left\|\lambda_{k}\right\|}\right\},
\end{gathered}
$$

then system (1) can reach consensus, and as $t \rightarrow \infty$,

$$
\begin{gathered}
X(t) \longrightarrow c(1,1, \ldots, 1)^{T} \\
V(t) \longrightarrow(0,0, \ldots, 0)^{T}
\end{gathered}
$$

where

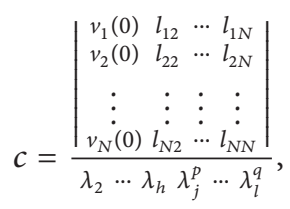

and $\lambda_{i}$ is the nonzero eigenvalue of the Laplacian matrix $L$. 
Journal of Applied Mathematics

3

Proof. Denoting $\mathscr{X}(s)=\mathscr{L}[X(t)]$, then (4) can be rewritten as

$$
\left[\left(s^{2}+s\right) I+e^{-s \tau} L\right] \mathscr{X}(s)=V(0) .
$$

According to Cramer's rule, we can have

$$
\mathscr{X}_{i}(s)=\frac{\operatorname{det}\left[\left(s^{2}+s\right) I+e^{-s \tau} L\right]^{i}}{\operatorname{det}\left[\left(s^{2}+s\right) I+e^{-s \tau} L\right]},
$$

for $i=1,2, \ldots, N$, where $\operatorname{det}\left[\left(s^{2}+s\right) I+e^{-s \tau} L\right]$ is the determinaut of matrix $\left[\left(s^{2}+s\right) I+e^{-s \tau} L\right]$, and $\operatorname{det}\left[\left(s^{2}+s\right) I+e^{-s \tau} L\right]^{i}$ is the determinant of matrix $\left[\left(s^{2}+s\right) I+e^{-s \tau} L\right]$ in which the $i$ th column has been replaced by $V(0)$. For convenience, we denote $M_{i}(s)=\operatorname{det}\left[\left(s^{2}+s\right) I+e^{-s \tau} L\right]^{i}$ and $M(s)=\operatorname{det}\left[\left(s^{2}+\right.\right.$ $\left.s) I+e^{-s \tau} L\right]$. By Lemma 1 , the Laplacian matrix only has a zero eigenvalue, and the real parts of nonzero eigenvalues are positive, denoted as follows:

$$
0, \lambda_{2}, \lambda_{3}, \ldots, \lambda_{h}, \lambda_{j}, \ldots, \lambda_{l}
$$

where $\lambda_{j}, \ldots, \lambda_{l}$ are the multiple eigenvalues with multiplicity as $p, \ldots, q$, then

$$
\begin{aligned}
M(s)= & {\left[\left(s^{2}+s\right)\right]\left[\left(s^{2}+s\right)+e^{-s \tau} \lambda_{2}\right] \cdots\left[\left(s^{2}+s\right)+e^{-s \tau} \lambda_{h}\right] } \\
& \times\left[\left(s^{2}+s\right)+e^{-s \tau} \lambda_{j}\right]^{p} \cdots\left[\left(s^{2}+s\right)+e^{-s \tau} \lambda_{l}\right]^{q} .
\end{aligned}
$$

Applying Heaviside's method, then

$$
\begin{aligned}
X_{i}(s)= & \frac{c_{1}^{i}}{\left(s^{2}+s\right)}+\frac{c_{2}^{i}}{\left(s^{2}+s\right)+e^{-s \tau} \lambda_{2}}+\cdots \\
& +\frac{c_{h}^{i}}{\left(s^{2}+s\right)+e^{-s \tau} \lambda_{h}}+\frac{c_{j p}^{i}}{\left[\left(s^{2}+s\right)+e^{-s \tau} \lambda_{j}\right]^{p}} \\
& +\frac{c_{j(p-1)}^{i}}{\left[\left(s^{2}+s\right)+e^{-s \tau} \lambda_{j}\right]^{(p-1)}}+\cdots \\
& +\frac{c_{j 1}^{i}}{\left[\left(s^{2}+s\right)+e^{-s \tau} \lambda_{j}\right]^{1}}+\cdots \\
& +\frac{c_{l q}^{i}}{\left[\left(s^{2}+s\right)+e^{-s \tau} \lambda_{l}\right]^{q}}+\frac{c_{l(q-1)}^{i}}{\left[\left(s^{2}+s\right)+e^{-s \tau} \lambda_{l}\right]^{(q-1)}} \\
& \left.+\cdots+\frac{c_{l 1}^{i}}{\left[\left(s^{2}+s\right)+e^{-s \tau}\right.} \lambda_{l}\right]^{1}
\end{aligned}
$$

where

$$
\begin{gathered}
c_{1}^{i}=\left.s \frac{M_{i}(s)}{M(s)}\right|_{s=0}, \\
c_{e}^{i}=\left.\left(s+\lambda_{e}\right) \frac{M_{i}(s)}{M(s)}\right|_{s=\lambda_{e}}, \quad e=2,3, \ldots, h, \\
c_{j f}^{i}=\left.\frac{d^{p-f}}{d s^{p-f}}\left[\left(s+\lambda_{j}\right)^{(p-f)} \frac{M_{i}(s)}{M(s)}\right]\right|_{s=\lambda_{j}}, \quad f=1,2, \ldots, p, \\
\vdots \quad \vdots \\
c_{k g}^{i}=\left.\frac{d^{q-g}}{d s^{q-g}}\left[\left(s+\lambda_{k}\right)^{(q-g)} \frac{M_{i}(s)}{M(s)}\right]\right|_{s=\lambda_{k}}, \quad g=1,2, \ldots, q .
\end{gathered}
$$

Therefore,

$$
\begin{aligned}
& \mathscr{X}_{i}(s) \geq \mathbf{G}_{i}(s) \\
& =\frac{c_{1}^{i}}{\left(s^{2}+s\right)}+\frac{c_{2}^{i}}{(s+1)^{2}+e^{-s \tau} \lambda_{2}} \\
& +\cdots+\frac{c_{h}^{i}}{(s+1)^{2}+e^{-s \tau} \lambda_{h}} \\
& +\frac{c_{j p}^{i}}{\left[(s+1)^{2}+e^{-s \tau} \lambda_{j}\right]^{p}}+\frac{c_{j(p-1)}^{i}}{\left[(s+1)^{2}+e^{-s \tau} \lambda_{j}\right]^{(p-1)}} \\
& +\cdots+\frac{c_{j 1}^{i}}{\left[(s+1)^{2}+e^{-s \tau} \lambda_{j}\right]^{1}} \\
& +\cdots+\frac{c_{l q}^{i}}{\left[(s+1)^{2}+e^{-s \tau} \lambda_{l}\right]^{q}} \\
& +\frac{c_{l(q-1)}^{i}}{\left[(s+1)^{2}+e^{-s \tau} \lambda_{l}\right]^{(q-1)}}+\cdots \\
& +\frac{c_{l 1}^{i}}{\left[(s+1)^{2}+e^{-s \tau} \lambda_{l}\right]^{1}} \\
& \mathscr{X}_{i}(s) \leq \mathbf{H}_{i}(s) \\
& =\frac{c_{1}^{i}}{\left(s^{2}+s\right)}+\frac{c_{2}^{i}}{s+e^{-s \tau} \lambda_{2}} \\
& +\cdots+\frac{c_{h}^{i}}{s+e^{-s \tau} \lambda_{h}} \\
& +\frac{c_{j p}^{i}}{\left[s+e^{-s \tau} \lambda_{j}\right]^{p}}+\frac{c_{j(p-1)}^{i}}{\left[s+e^{-s \tau} \lambda_{j}\right]^{(p-1)}}
\end{aligned}
$$




$$
\begin{aligned}
& +\cdots+\frac{c_{j 1}^{i}}{\left[s+e^{-s \tau} \lambda_{j}\right]^{1}} \\
& +\cdots+\frac{c_{l q}^{i}}{\left[s+e^{-s \tau} \lambda_{l}\right]^{q}} \\
& +\frac{c_{l(q-1)}^{i}}{\left[s+e^{-s \tau} \lambda_{l}\right]^{(q-1)}}+\cdots \\
& +\frac{c_{l 1}^{i}}{\left[s+e^{-s \tau} \lambda_{l}\right]^{1}} .
\end{aligned}
$$

In order to make $\mathbf{G}_{i}(s)$ converge, the real parts of the following equation's roots

$$
(s+1)^{2}+e^{-s \tau} \lambda_{k}=0
$$

must be negative. Denoting $s=x+j y, \lambda_{k}=\alpha_{k}+j \beta_{k}$, and $j^{2}=-1$, as well as using Euler's formula, then

$$
\begin{aligned}
& {\left[(x+1)^{2}-y^{2}+\alpha_{k} e^{-x \tau} \cos (-y \tau)-\beta_{k} e^{-x \tau} \sin (-y \tau)\right]} \\
& \quad+j\left[2(x+1) y+\alpha_{k} e^{-x \tau} \sin (-y \tau)-\beta_{k} e^{-x \tau} \cos (-y \tau)\right] \\
& \quad=0
\end{aligned}
$$

that is,

$$
\begin{gathered}
(x+1)^{2}-y^{2}+\alpha_{k} e^{-x \tau} \cos (-y \tau)-\beta_{k} e^{-x \tau} \sin (-y \tau)=0 \\
2(x+1) y+\alpha_{k} e^{-x \tau} \sin (-y \tau)-\beta_{k} e^{-x \tau} \cos (-y \tau)=0 .
\end{gathered}
$$

And then

$$
\begin{gathered}
(x+1)^{2}-y^{2}+e^{-x \tau} \sqrt{\alpha_{k}^{2}+\beta_{k}^{2}} \cos \left(-y \tau+\psi_{k}\right)=0 \\
2(x+1) y+e^{-x \tau} \sqrt{\alpha_{k}^{2}+\beta_{k}^{2}} \sin \left(-y \tau+\psi_{k}\right)=0,
\end{gathered}
$$

where $\psi_{k}=\arg \left(\lambda_{k}\right)=\arctan \left(\beta_{k} / \alpha_{k}\right)$ and $\psi_{k} \in(-\pi / 2, \pi / 2)$. Then we can have

$$
\left((x+1)^{2}+y^{2}\right)^{2}=\left(\alpha_{k}^{2}+\beta_{k}^{2}\right) e^{-2 x \tau}
$$

that is,

$$
y= \pm \sqrt{\sqrt{\alpha_{k}^{2}+\beta_{k}^{2}} e^{-x \tau}-(x+1)^{2}} .
$$

Form (18) and (20), we can obtain

$$
\begin{aligned}
& 2(x+1)^{2}-\sqrt{\alpha_{k}^{2}+\beta_{k}^{2}} e^{-x \tau}+\sqrt{\alpha_{k}^{2}+\beta_{k}^{2}} e^{-x \tau} \\
& \times \cos \left( \pm \sqrt{\sqrt{\alpha_{k}^{2}+\beta_{k}^{2}} e^{-x \tau}-(x+1)^{2}}+\psi_{k}\right)=0, \\
& 2(x+1)\left( \pm \sqrt{\sqrt{\alpha_{k}^{2}+\beta_{k}^{2}} e^{-x \tau}-(x+1)^{2}}\right)+\sqrt{\alpha_{k}^{2}+\beta_{k}^{2}} e^{-x \tau} \\
& \times \sin \left( \pm \tau \sqrt{\sqrt{\alpha_{k}^{2}+\beta_{k}^{2}} e^{-x \tau}-(x+1)^{2}}+\psi_{k}\right)=0 .
\end{aligned}
$$

Considering the case $x=0$, we can obtain the upper bound of time-delay

$$
\cos \left(\tau \sqrt{\sqrt{\alpha_{k}^{2}+\beta_{k}^{2}}-1} \pm \psi_{k}\right)=\frac{\sqrt{\alpha_{k}^{2}+\beta_{k}^{2}}-2}{\sqrt{\alpha_{k}^{2}+\beta_{k}^{2}}}
$$

that is,

$$
\begin{array}{r}
\tau \sqrt{\left\|\lambda_{k}\right\|-1} \pm \arg \left(\lambda_{k}\right)=\arccos \left(\frac{\left\|\lambda_{k}\right\|-2}{\left\|\lambda_{k}\right\|}\right)+2 m \pi \\
m=0, \pm 1, \pm 2, \ldots .
\end{array}
$$

To obtain the minimum critical value $\tau_{1}$, we take $m=0$, then

$$
\tau_{1}=\min _{k=\{2,3, \ldots, N\}}\left\{\left|\frac{\arccos \left(\left(\left\|\lambda_{k}\right\|-2\right) /\left\|\lambda_{k}\right\|\right)-\arg \left(\lambda_{k}\right)}{\sqrt{\left\|\lambda_{k}\right\|-1}}\right|\right\} .
$$

Then we can obtain the real parts of roots of $(s+1)^{2}+e^{-s \tau} \lambda_{k}=$ 0 are negative as $\tau<\tau_{1}$.

Similarly, for $\mathbf{H}_{i}(s)$, we can also get the upper bound of time-delay $\tau_{2}$; that is, for

$$
s+\lambda_{k} e^{-s \tau}=0
$$

the real parts of roots are negative. So

$$
\tau_{2}=\min _{k=\{2,3, \ldots, N\}}\left\{\frac{\pi / 2-\left|\arg \left(\lambda_{k}\right)\right|}{\left\|\lambda_{k}\right\|}\right\} .
$$

When

$$
\tau<\min _{k=\{2,3, \ldots, N\}}\left\{\frac{\left|\arccos \left(\left(\left\|\lambda_{k}\right\|-2\right) /\left\|\lambda_{k}\right\|\right)-\arg \left(\lambda_{k}\right)\right|}{\sqrt{\left\|\lambda_{k}\right\|-1}},\right.
$$

$$
\left.\frac{\pi / 2-\left|\arg \left(\lambda_{k}\right)\right|}{\left\|\lambda_{k}\right\|}\right\}
$$




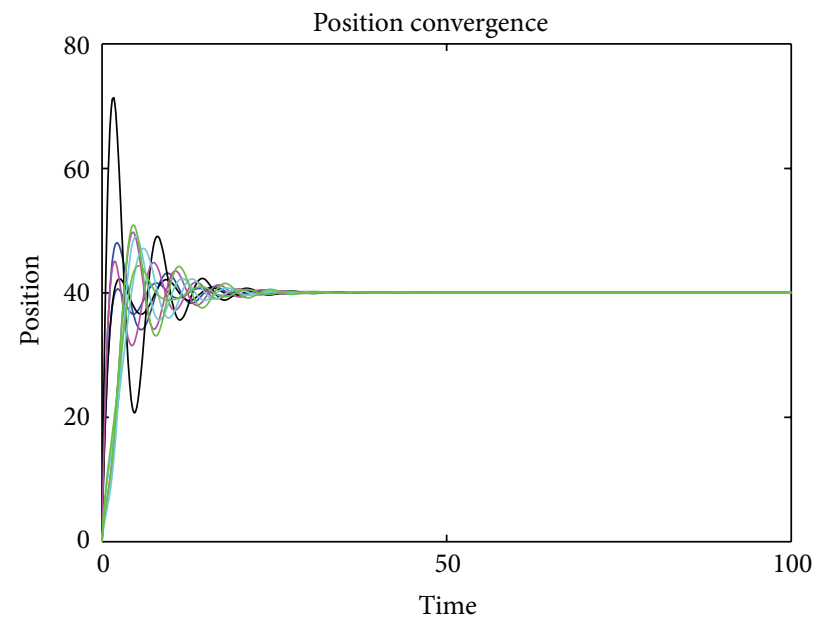

(a)

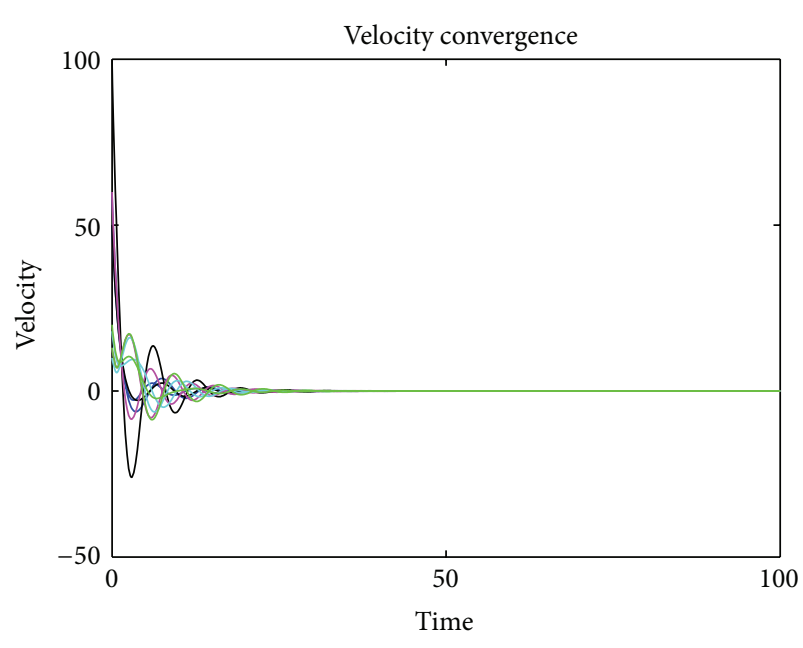

(b)

FIGURE 1: The consensus in system (1) with the general topology as $\tau=0.5$.

then the roots of equations $(s+1)^{2}+e^{-s \tau} \lambda_{k}=0$ and $s+\lambda_{k} e^{-s \tau}=$ 0 are negative real parts.

By the inverse Laplace transform, we can obtain

$$
\mathscr{L}^{-1}\left(\mathbf{G}_{i}(s)\right) \longrightarrow c_{1}^{i}, \quad \mathscr{L}^{-1}\left(\mathbf{H}_{i}(s)\right) \longrightarrow c_{1}^{i},
$$

as $t \rightarrow \infty$.

Since $\mathbf{G}_{i}(s) \leq \mathscr{X}_{i}(s) \leq \mathbf{H}_{i}(s)$, as $t \rightarrow \infty$

$$
\mathscr{L}^{-1}\left(\mathscr{X}_{i}(s)\right) \longrightarrow c_{1}^{i} \text {. }
$$

According to Lemma 3, we can know that

$$
\begin{aligned}
& c_{1}^{1}=c_{1}^{2}=\cdots=c_{1}^{N}=\left.s \frac{M_{i}(s)}{M(s)}\right|_{s=0}
\end{aligned}
$$

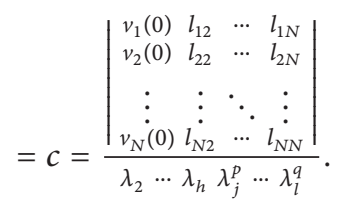

Thus,

$$
\begin{aligned}
& x_{1}(t)=x_{2}(t)=\cdots=x_{N}(t)=c, \\
& v_{1}(t)=v_{2}(t)=\cdots=v_{N}(t)=0 ;
\end{aligned}
$$

that is, as $t \rightarrow \infty$,

$$
\begin{gathered}
X(t) \longrightarrow c(1,1, \ldots, 1)^{T}, \\
V(t) \longrightarrow(0,0, \ldots, 0)^{T} .
\end{gathered}
$$

This completes the proof.

Notice that if the network topology is undirected with a globally reachable node and the adjacency matrix is symmetric, then the eigenvalues of the Laplacian matrix $L$ are real, so that $\psi_{k}=0$.
Remark 5. Compared with the eigenvalue analysis in the time and frequency domain, such as the [24], using Cramer rule and Heavisides method, we can have obtained the accurate state of the consensus center and the upper bound of the communication delay to make the agents reach consensus.

Corollary 6. For second-order multi-agent system (1), if there exists at least one globally reachable node in the undirected and symmetric network topology, and the time-delay $\tau$ satisfies

$$
\begin{gathered}
\tau<\min _{k=\{2,3, \ldots, N\}}\left\{\frac{\left|\arccos \left(\left(\left\|\lambda_{k}\right\|-2\right) /\left\|\lambda_{k}\right\|\right)\right|}{\sqrt{\left\|\lambda_{k}\right\|-1}}\right. \\
\left.\frac{\pi}{2\left\|\lambda_{k}\right\|}\right\},
\end{gathered}
$$

then system (1) can reach consensus.

3.1. Further Extension. Consider the second-order multiagent system with time-delay and general topology is described by

$$
\begin{gathered}
\dot{x}_{i}=v_{i} \\
\dot{v}_{i}=-\sum_{j \in \mathcal{N}_{i}} a_{i j}\left(x_{i}(t-\tau)-x_{j}(t-\tau)\right)-\gamma v_{i},
\end{gathered}
$$

where $\gamma>0$ is the feedback gain.

By the same way, the similar results are easy to obtain by the Laplace transform. 


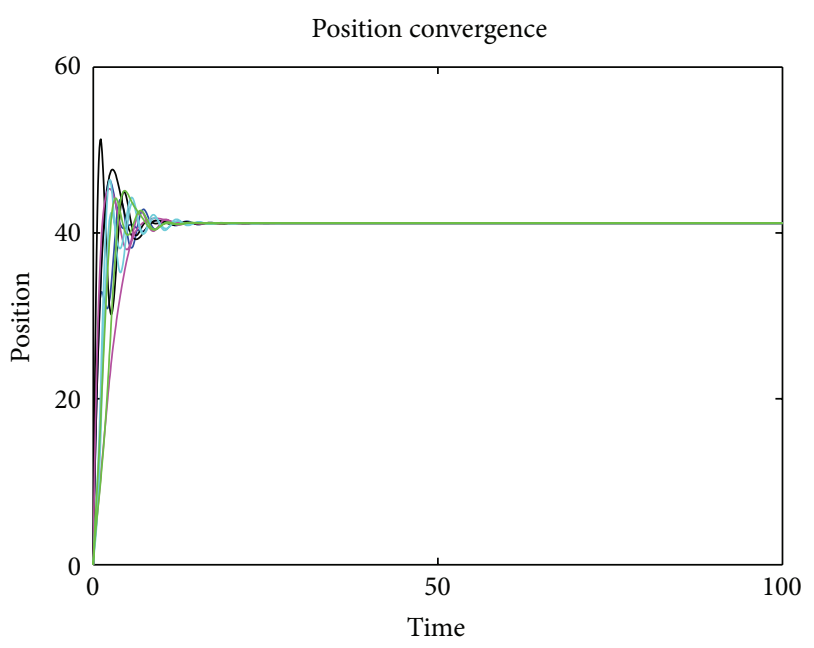

(a)

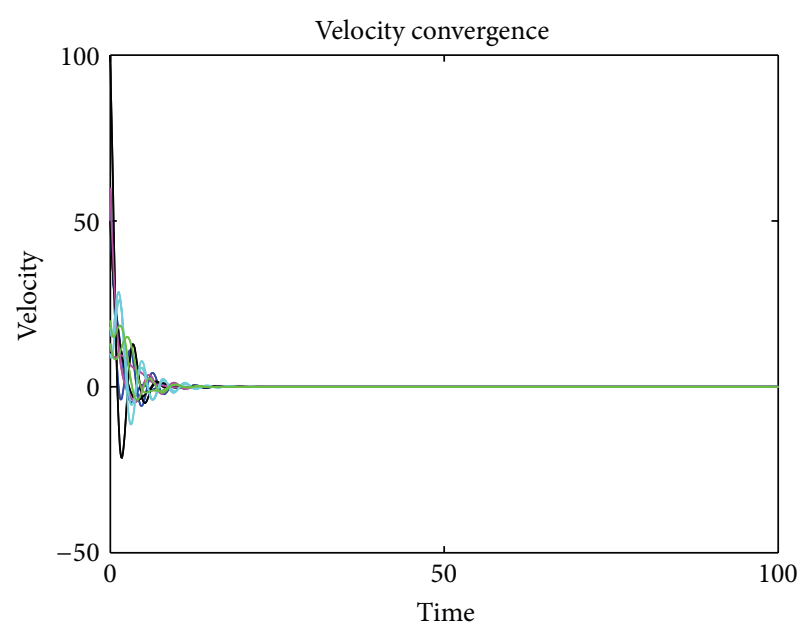

(b)

FIGURE 2: The consensus in system (1) with the symmetric topology as $\tau=0.1$.

Theorem 7. For second-order multi-agent system (35), if there exists at least one globally reachable node in the network topology, and the time-delay $\tau$ satisfies

$$
\begin{gathered}
\tau<\min _{k=\{2,3, \ldots, N\}}\left\{\frac{\left|\arccos \left(\left(\left\|\lambda_{k}\right\|-2 \gamma^{2}\right) /\left\|\lambda_{k}\right\|\right)-\arg \left(\lambda_{k}\right)\right|}{\sqrt{\left\|\lambda_{k}\right\|-\gamma^{2}}},\right. \\
\left.\frac{\gamma\left(\pi / 2-\left|\arg \left(\lambda_{k}\right)\right|\right)}{\left\|\lambda_{k}\right\|}\right\},
\end{gathered}
$$

then system (35) can reach consensus, and as $t \rightarrow \infty$,

$$
X(t) \longrightarrow c(1,1, \ldots, 1)^{T}, \quad V(t) \longrightarrow(0,0, \ldots, 0)^{T}
$$

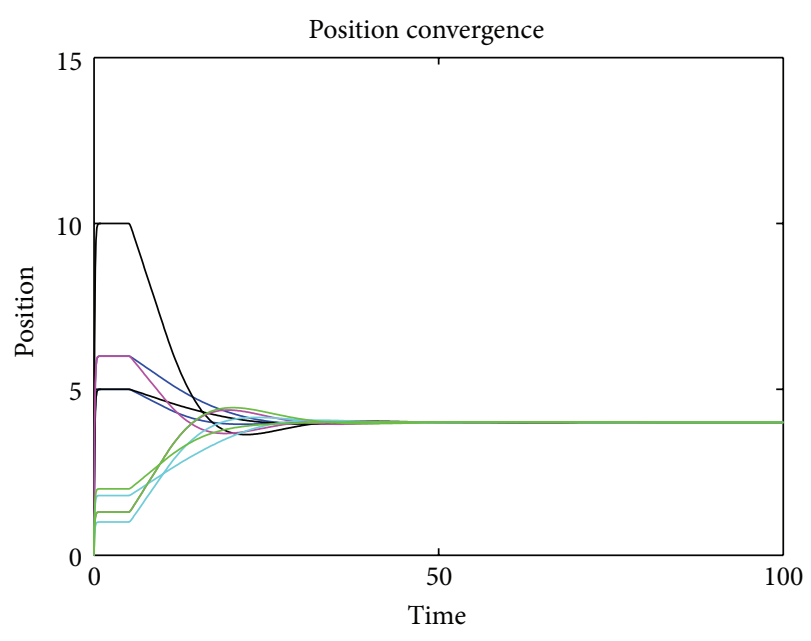

(a)

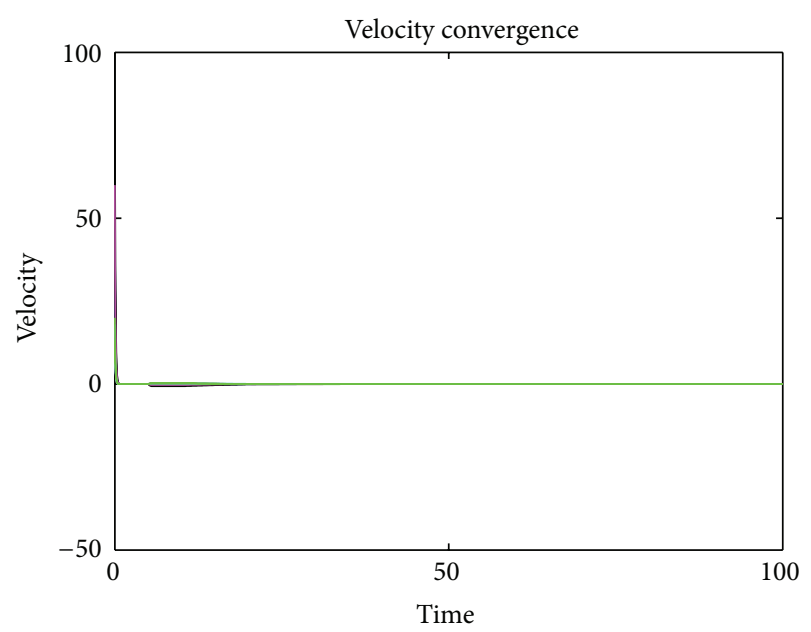

(b)

FIGURE 3: The consensus in system (35) with the general topology as $\tau=5, \gamma=10$.

Corollary 8. For second-order multi-agent system (35), if there exists at least one globally reachable node in the undirected and symmetric network topology, and the time-delay $\tau$ satisfies

$$
\tau<\min _{k=\{2,3, \ldots, N\}}\left\{\frac{\left|\arccos \left(\left(\left\|\lambda_{k}\right\|-2 \gamma^{2}\right) /\left\|\lambda_{k}\right\|\right)\right|}{\sqrt{\left\|\lambda_{k}\right\|-\gamma^{2}}}, \frac{\gamma \pi}{2\left\|\lambda_{k}\right\|}\right\},
$$

then system (35) can reach consensus.

\section{Numerical Simulations}

In this section, we present some numerical simulations to illustrate the consensus of second-order multi-agent systems with time delay. These simulations are given with ten agents, whose initial positions are zero and velocities are given 


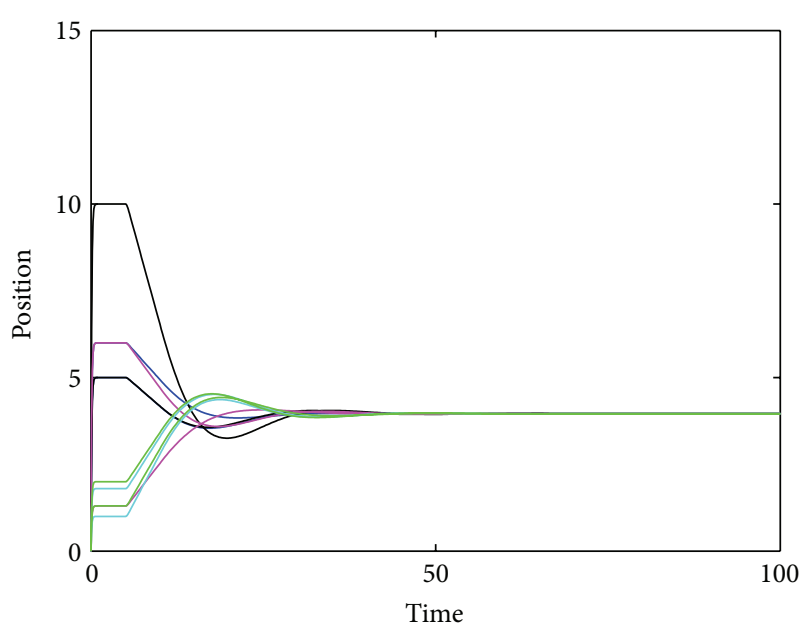

(a)

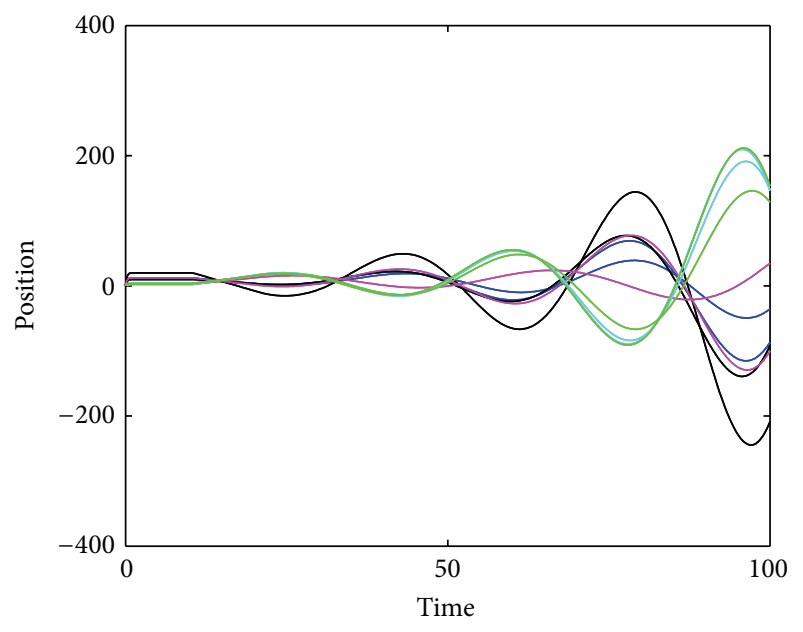

(b)

FIgURE 4: The position states in system (35) with the symmetric topology as $\tau=5, \gamma=10$ and $\tau=10, \gamma=5$.

randomly. The network topologies contain at least one globally reachable node in these simulations. Figure 1 describes positions and velocities of ten agents in system (1) with the general topology. From Figure 1, it can be seen that the positions and velocities of all agents can reach consensus as $\tau=0.5$.

Figure 2 shows that positions and velocities can converge to the common value in system (1) with symmetrical matrix as $\tau=0.1$.

In Figure 3, the consensus can be achieved in system (35) with the general topology as $\tau=5, \gamma=10$. As we can see from Figures 1-3, the consensus of systems can be achieved when the time-delay is an appropriate value. The positions of all agent converge on a fixed value and velocities reach 0 .

Figure 4 presents that the positions of all agents with the general topology can reach consensus as $\tau=5, \gamma=10$, while the positions diverge as $\tau=10, \gamma=5$. From Figure 4, it can be seen that the system can reach consensus under a certain condition. According to the numerical simulations, we can obtain that the topology of network topology, time-delay, and feedback gain all affect consensus of multi-agent system in the simulations.

\section{Conclusion}

In this paper, we have introduced the Laplace transform to investigate the consensus of second-order multi-agent system with time-delay in general topology. We have proved that the system can achieve consensus when the network topology contains a globally reachable node as well as when time-delay is less than a critical value which only depends on eigenvalues of the Laplacian matrix of network topology.

\section{Acknowledgments}

The authors would like to thank the associate editor and the anonymous reviewer for their valuable comments and suggestions, which significantly contributed to improving the quality of the paper. This work was supported by the National Natural Science Foundation of China under Grants nos. 60774089, 10972003, and 61203150, the Beijing Natural Science Foundation Program (1102016, 4122019), Science and Technology Development Plan Project of Beijing Education Commission (no. KM201310009011); and the Funding Project for Academic Human Resources Development in Institutions of Higher Learning under the Jurisdiction of Beijing Municipality (PHR201108055). This work was also supported by the Foundation Grant of Guangxi Key Laboratory of Automobile Components and Vehicle Technology (13-A-03-01), the Opening Project of Guangxi Key Laboratory of Automobile Components and Vehicle Technology (2012KFZD03).

\section{References}

[1] B. Liu, X. Zhu, and J. Zhang, "Consensus analyss of the multiagent sysem," in Proceedings of the 3rd IEEE International Workshop on Chaos-Fractals Theories and Applications (IWCFTA '10), pp. 383-387, October 2010.

[2] Y. G. Sun, L. Wang, and G. Xie, "Average consensus in networks of dynamic agents with switching topologies and multiple timevarying delays," Systems \& Control Letters, vol. 57, no. 2, pp. 175$183,2008$.

[3] Y. Hong, G. Chen, and L. Bushnell, "Distributed observers design for leader-following control of multi-agent networks," Automatica, vol. 44, no. 3, pp. 846-850, 2008.

[4] R. Olfati-Saber and R. M. Murray, "Consensus problems in networks of agents with switching topology and time-delays," IEEE Transactions on Automatic Control, vol. 49, no. 9, pp. 15201533,2004

[5] R. Olfati-Saber, J. A. Fax, and R. M. Murray, "Consensus and cooperation in networked multi-agent systems," Proceedings of the IEEE, vol. 95, no. 1, pp. 215-233, 2007.

[6] W. Yu, G. Chen, and M. Cao, "On second-order consensus in multi-agent dynamical systems with directed topologies and time delays," in Proceedings of the 48th IEEE Conference on Decision and Control held jointly with the 28th Chinese Control Conference (CDC/CCC '09), pp. 3709-3714, December 2009. 
[7] W. Ren, "Collective motion from consensus with Cartesian coordinate coupling," IEEE Transactions on Automatic Control, vol. 54, no. 6, pp. 1330-1335, 2009.

[8] G. Xie and L. Wang, "Consensus control for a class of networks of dynamic agents: fixed topology," in Proceedings of the 44th IEEE Conference on Decision and Control, and the European Control Conference (CDC-ECC '05), pp. 96-101, December 2005.

[9] J. Hu and Y. Hong, "Leader-following coordination of multiagent systems with coupling time delays," Physica A, vol. 374, no. 2, pp. 853-863, 2007.

[10] T. Vicsek and A. Czirok, "Novel type of phase transitions in a system of self-driven," Physical Review Letters, vol. 75, pp. 12261229, 1995.

[11] A. Jadbabaie, J. Lin, and A. S. Morse, "Coordination of groups of mobile autonomous agents using nearest neighbor rules," IEEE Transactions on Automatic Control, vol. 48, no. 6, pp. 988-1001, 2003.

[12] Y. Gao and L. Wang, "Sampled-data based consensus of continuous-time multi-agent systems with time-varying topology," IEEE Transactions on Automatic Control, vol. 56, no. 5, pp. 1226-1231, 2011.

[13] W. Ren, "On consensus algorithms for double-integrator dynamics," IEEE Transactions on Automatic Control, vol. 53, no. 6, pp. 1503-1509, 2008.

[14] M. H. DeGroot, "Reach a consensus," Journal of American Statistical Association, vol. 69, no. 345, pp. 118-121, 1974.

[15] W. Yu, Z. Wang, and W. Yang, "Distributed consensus filtering in sensor networks," IEEE Transactions on Systems, Man, and Cybernetics B, vol. 39, no. 6, pp. 1568-1577, 2009.

[16] W. Yu, W. X. Zheng, G. Chen, W. Ren, and J. Cao, "Second-order consensus in multi-agent dynamical systems with sampled position data," Automatica, vol. 47, no. 7, pp. 1496-1503, 2011.

[17] W. Ren, "On consensus algorithms for double-integrator dynamics," in Proceedings of the 46th IEEE Conference on Decision and Control, pp. 2295-2300, 2007.

[18] W. Ren and E. Atkins, "Second-order consensus protocols in multiple vehicle systems with local interactions," in Proceedings of the AIAA Guidance, Navigation, and Control Conference, pp. 3689-3701, August 2005.

[19] W. Yu, G. Chen, and M. Cao, "Some necessary and sufficient conditions for second-order consensus in multi-agent dynamical systems," Automatica, vol. 46, no. 6, pp. 1089-1095, 2010.

[20] B. Liu, T. Chu, L. Wang, and Z. Wang, "Collective motion of a class of social foraging swarms," Chaos, Solitons and Fractals, vol. 38, no. 1, pp. 277-292, 2008.

[21] B. Liu, T. Chu, and L. Wang, "Stability and oscillation of swarm with interaction time delays," in Proceedings of the American Control Conference (ACC '07), pp. 4600-4605, July 2007.

[22] P. Lin and Y. Jia, "Consensus of a class of second-order multiagent systems with time-delay and jointly-connected topologies," IEEE Transactions on Automatic Control, vol. 55, no. 3, pp. 778-784, 2010.

[23] B. Liu, X. Zhu, J. Zhang, and Y. Sun, "Consensus of multi-agent systems based on leader-following control," in Proceedings of the 6th International Conference on Natural Computation (ICNC '10), pp. 1366-1370, August 2010.

[24] W. Yu, G. Chen, M. Cao, and J. Kurths, "Second-Order consensus for multiagent systems with directed topologies and nonlinear dynamics," IEEE Transactions on Systems, Man, and Cybernetics B, vol. 40, no. 3, pp. 881-891, 2010.
[25] Y. Cao, W. Yu, W. Ren, and G. Chen, "An overview of recent progress in the study of distributed multi-agent coordination," IEEE Transactions on Industrial Informatics, vol. 9, no. 1, pp. 427-438, 2013. 


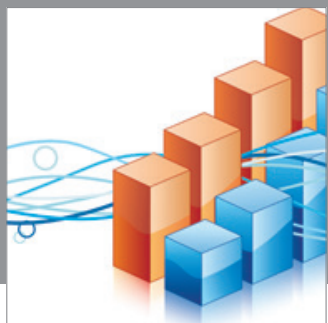

Advances in

Operations Research

mansans

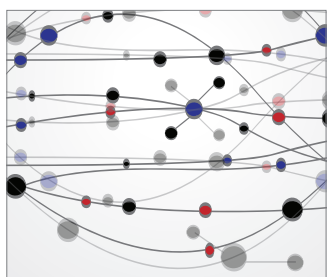

The Scientific World Journal
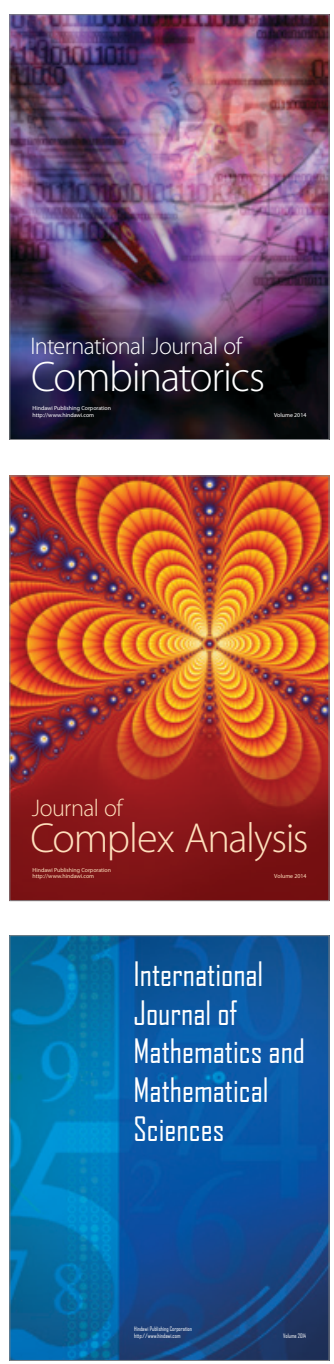
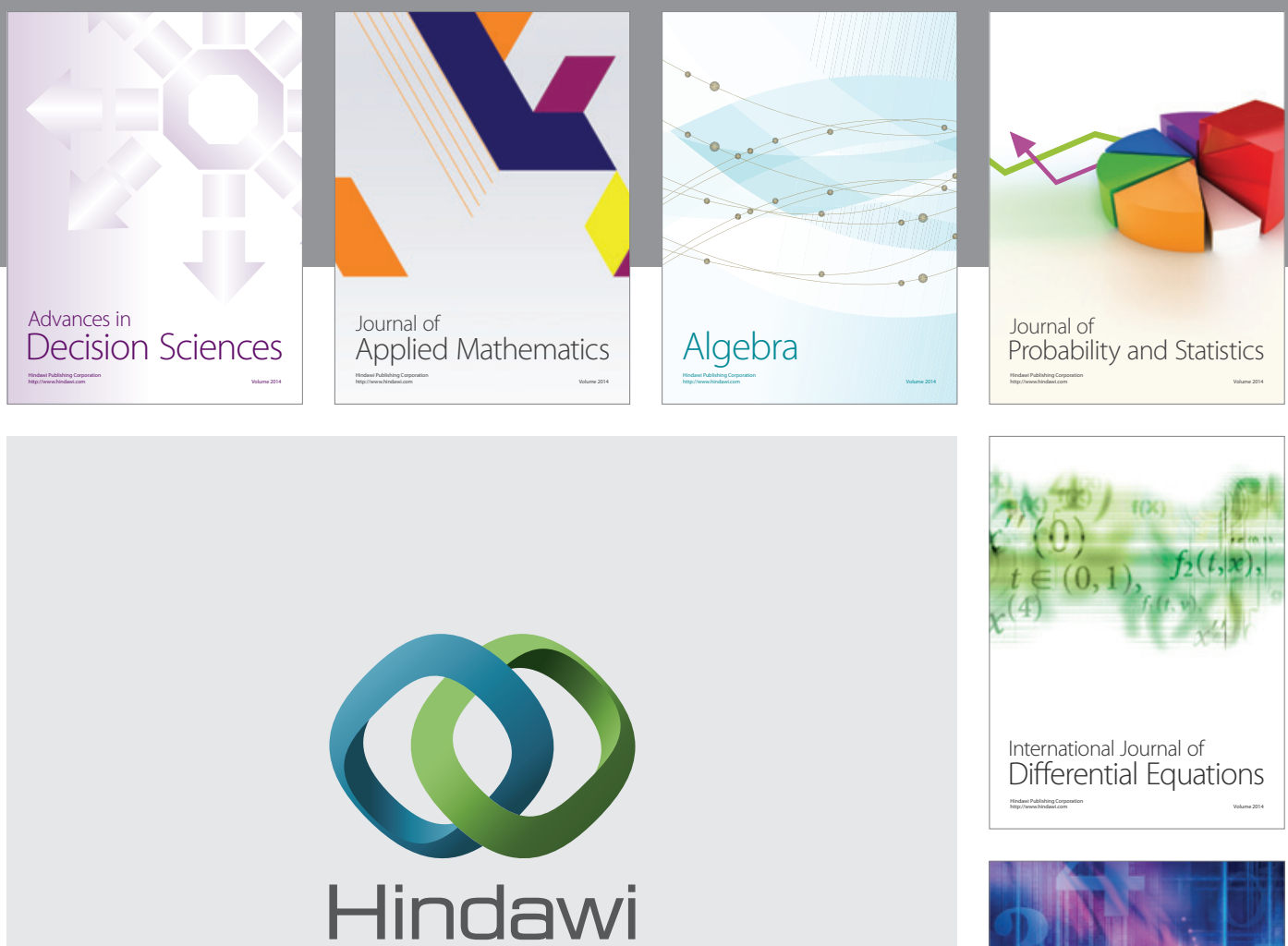

Submit your manuscripts at http://www.hindawi.com
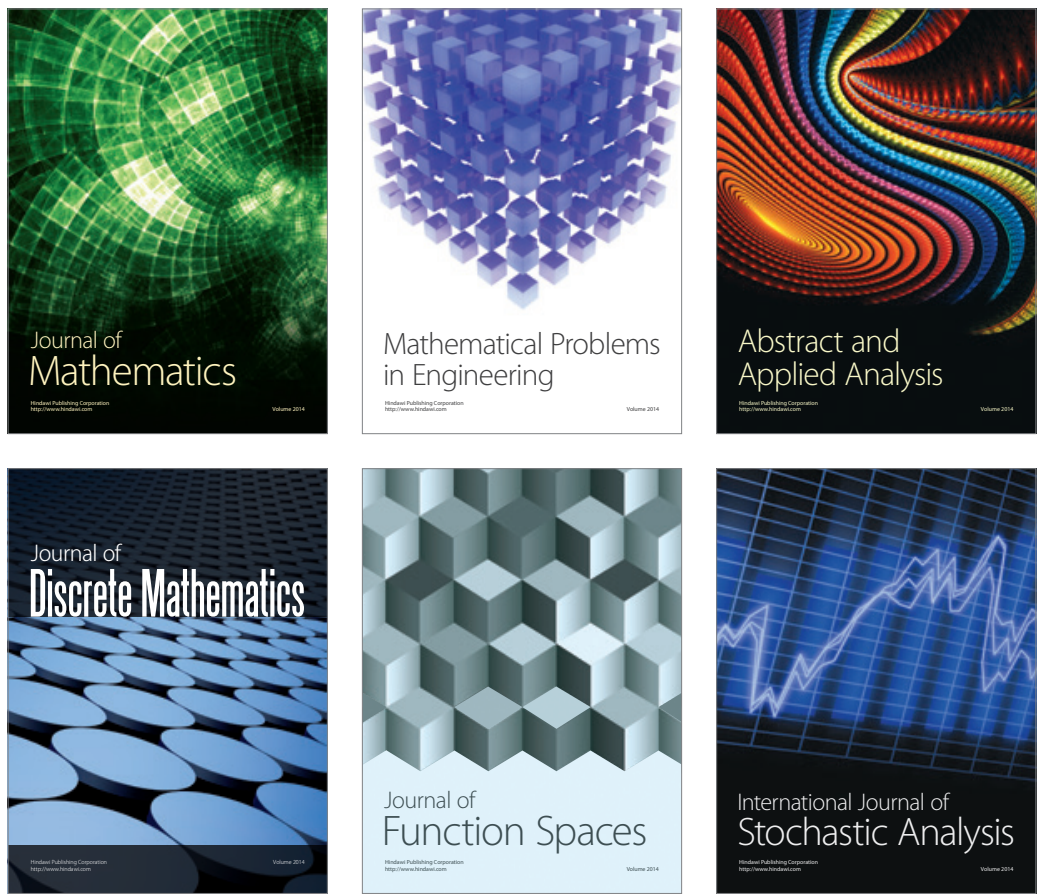

Journal of

Function Spaces

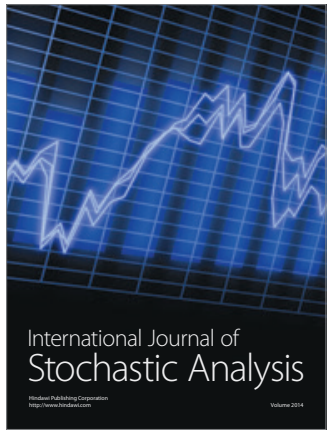

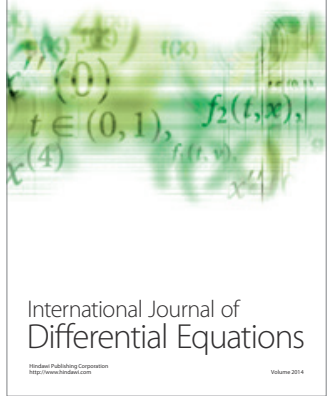
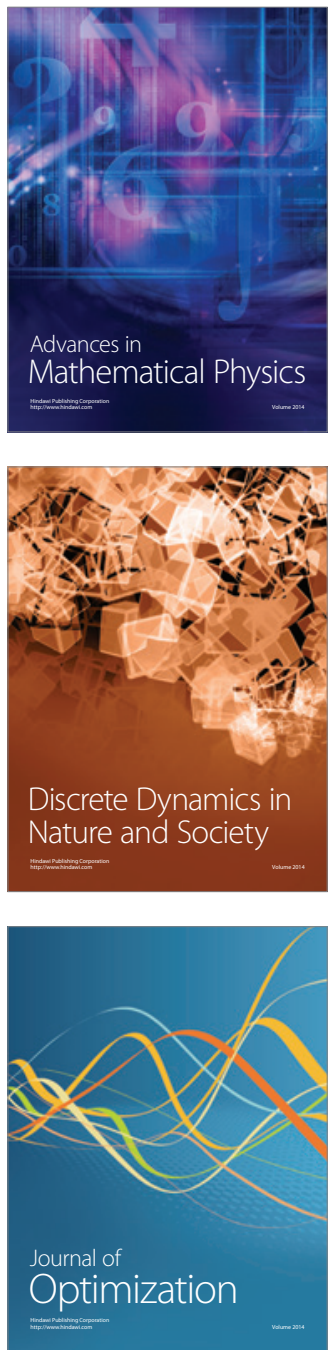